\title{
Polymer-Encapsulated Metallic Nanoparticles as a Bridge Between Homogeneous and Heterogeneous Catalysis
}

\author{
Elad Gross • F. Dean Toste • Gabor A. Somorjai
}

Received: 6 October 2014/ Accepted: 13 November 2014/Published online: 25 November 2014

(c) Springer Science+Business Media New York 2014

\begin{abstract}
Continuous efforts in catalysis research have been devoted towards the development of heterogeneous catalysts that can activate reactions which are catalyzed by homogeneous catalysts. Replacing homogeneous catalysts with their heterogeneous counterparts will enhance the sustainability of the catalytic system, providing a highly recyclable, scalable and efficient setup. Throughout this review we demonstrate that small $(<2 \mathrm{~nm})$, metallic nanoclusters can catalyze a wide range of $\pi$-bond activation reactions that were previously activated by homogeneous catalysts. The small size of the nanoparticles enables their reversible oxidation into catalytically active metal ions. Encapsulation of the metal within a polymeric matrix severely restricts leaching of the highly oxidized metal ions into the solution phase, inducing high catalytic stability and recyclability. Activation of complex, multistep organic transformations with heterogeneous catalysts provides novel opportunities, not accessible with homogeneous catalysts, to control and tune the products selectivity. By designing the molecular properties of the polymeric matrix that encapsulates the metal cluster, high products selectivity, diastereoselectivity and enantiose-
\end{abstract}

E. Gross $(\bowtie) \cdot$ F. Dean Toste · G. A. Somorjai

Department of Chemistry, University of California,

Berkeley 94720, CA, USA

e-mail: elad.gross@mail.huji.ac.il

E. Gross · F. Dean Toste · G. A. Somorjai

Chemical and Materials Science Divisions, Lawrence Berkeley

National Laboratory, Berkeley 94720, CA, USA

Present Address:

E. Gross

Institute of Chemistry, The Hebrew University of Jerusalem,

Jerusalem, Israel lectivity can be gained. These results demonstrate the capability of mesoscale catalysts, constructed of metallic nanoparticles and an encapsulating layer, to activate a wide array of catalytic reactions with high reactivity and tunable selectivity.

Keywords Heterogeneous catalysis - Homogeneous catalysis - Asymmetric catalysis - Cyclization - Infrared tomography · NEXAFS · Colloidal synthesis

\section{Introduction}

Catalysis is a critical science for the modern society and improvements in catalytic processes will increase efficiencies of chemical transformations while reducing waste and environmental footprints. Highly selective catalysts, especially those that can be readily recycled, are vital for the development of sustainable chemical processes that can address the energy-related challenges of the 21 st century [1-3]. Therefore, the development of modular catalytic systems, in which different parameters within the catalyst can be tuned for optimization of its catalytic properties and reaction scope, are highly desirable.

The variability of homogeneous catalysts, constructed of a metal-ion core and surrounding ancillary ligands, enables the tuning of the catalyst's structure for optimization of its reactivity and selectivity $[4,5]$. For example, in homogenous catalysts, the steric and electronic features of the ancillary ligands are commonly varied in order to attain high stereoselectivity. In addition to the ligands, the ionic properties of the metal play a fundamental role during the catalytic process, since the catalytic reactivity is governed by metal-reactant interactions that are related to the oxidation state of the metal [6]. 
Heterogeneous catalysts, on the other hand, lack the high tunability of homogeneous catalysts but have the advantages of being readily recyclable and easily adopted in a fixed-bed flow reactor. The robustness, stability and recyclability of heterogeneous catalysts under harsh conditions makes them ideal catalysts for cleavage of strong bonds, such as $\mathrm{C}-\mathrm{O}$ and $\mathrm{C}-\mathrm{H}$ bonds. However, heterogeneous catalysts lack the high products selectivity (e.g. diastereoselectivity, enantioselectivity) which is routinely obtained while employing architecturally-designed organometallic complexes as homogeneous catalysts. The catalytic reactivity of heterogeneous catalysts can be moderately optimized by adjusting the nanoparticle's size, shape and the support on which it is deposited [7-10]. The limited reaction scope of heterogeneous catalysts emphasizes the lack of novel catalytic systems that can combine the recyclability of heterogeneous catalysts along with the wide reaction scope and high selectivity of homogeneous catalysts [11].

Continuous efforts have been devoted to activate heterogeneous catalysts toward reactions that are mainly performed with homogeneous catalysts [12]. The conventional method of heterogenizing homogeneous catalysts relies on grafting highly selective transition metal complex catalysts onto mesoporous solid supports. It has been demonstrated that the supported metal sites can catalyze a variety of reactions, such as olefin methathesis and Ziegler-Natta depolymerization, with high reactivity and recyclability [13].

Another alternative for heterogenizing homogeneous catalysts is to directly embed homogenous catalysts within the structure of metal-organic frameworks. Highly selective solid catalysts were prepared by encapsulating catalyticallyactive sites within well-defined frameworks, which have specific pore dimensions and topologies [14-17]. For example, it was recently demonstrated that asymmetric hetero-Diels-Alder reactions can be activated by embedding catalytically active Ti(IV) ions within NbO-type chiral metal-organic frameworks [18]. A more challenging approach is to utilize the truly heterogeneous metallic nanoparticles as catalysts for reactions that are activated by homogeneous catalysts. In the recent years a variety of metallic nanoparticles, such as Au [19-29], Pt [30, 31], and $\mathrm{Pd}$ [32] have been employed as catalysts for complex organic transformations with high yield and selectivity. Spectroscopic measurements have indicated that the heterogeneous catalysts have to be partially charged prior to their activation as catalysts toward organic transformations. Two main approaches were taken in order to change the electronic properties of heterogeneous catalysts:

(i) In the first approach the metallic nanoparticles are charged following their deposition on an acidic or basic metal-oxide support [33, 34]. For example, it was demonstrated that the deposition of $\mathrm{Au}$ nanoparticles on $\mathrm{CeO}_{2}$ support facilitates the oxidation of the metallic $\left(\mathrm{Au}^{0}\right)$ nanoparticles into $\mathrm{Au}^{+3}$. X-ray spectroscopy measurements correlated between the oxidation state of the metal and its reactivity toward coupling reactions that were previously activated by $\mathrm{Au}$ ions [29-32].

(ii) In the second approach, the oxidation state of the nanoparticles and as a consequence also their catalytic reactivity, are tuned by controlling the size of the nanoparticles. It was previously demonstrated that there is a direct correlation between the nanoparticle's size and its electronic properties $[35,36]$. The oxidation state of nanoparticles can be transformed from metallic into ionic by decreasing the nanoparticles size below $2 \mathrm{~nm}$. The formation of small, highly oxidized nanoparticles with ionic properties change the catalytic properties of the metal and activate the clusters toward a variety of reactions which were previously catalyzed by homogeneous catalysts [37].

The partial oxidation of the metallic nanoparticle transforms the properties of the heterogeneous catalyst, making it similar to the metal ion core of homogeneous catalysts. However, the high selectivity of homogeneous catalysts is mainly gained by the ligands which surround the metallic core. These directing ligands are not present within heterogeneous catalysts. As a consequence, only in few cases heterogeneous catalysts showed similar products selectivity to that obtained by their homogeneous analogues [38].

In the following review paper we describe our recent work in heterogenizing homogeneous catalysts. We demonstrate that by tuning the properties of metallic nanoparticles and embedding the nanoparticles in a specificallydesigned polymeric matrix, the heterogeneous catalyst can be activated to promote a variety of organic transformations. The polymeric matrix that encapsulates the metallic nanoparticle has two main roles:

(i) The matrix stabilizes the small, highly oxidized, metallic nanoparticles and severely restricts their decomposition and leaching into the solution phase.

(ii) The encapsulating polymer also acts as a cocatalyst and its properties can be tuned in order to direct the reactivity and selectivity of the metal core.

In order to analyze the impact of the nanoparticle's oxidation state and its surrounding environment on the catalytic reactivity, a variety of in situ spectroscopy measurements have been conducted under reaction conditions 
[39-42]. Synchrotron based X-ray spectroscopy measurements have been performed in order to correlate between the oxidation state of the catalyst and its reactivity. In addition, synchrotron based FTIR microspectroscopy measurements tracked the reactants-into-products transformation and detected the role of different intermediates in the catalytic process.

\section{Results}

\subsection{Dendrimer-Templated Synthesis of Small $(\sim 1 \mathrm{~nm})$ Metallic Nanoparticles}

Metal nanoparticles in the size range of $10 \mathrm{~nm}$ and above have been widely synthesized by colloid techniques [7-10]. However, the synthesis of nanoparticles with size below $1 \mathrm{~nm}$ (tens of atoms) is more challenging. At this size regime, more than $90 \%$ of the atoms are located on the surface of the clusters. The atoms coordination number in these clusters is smaller than that of larger nanoparticles that have a substantial fraction of their atoms in the bulk. At sizes smaller than $1 \mathrm{~nm}$, the nanoparticles have shorter metal-metal bonds, lower melting points, and are easier to oxidize than larger particles. These properties also impact the catalytic reactivity of the nanoparticles [42].

A dendrimer templating strategy is very attractive for synthesis of small nanoparticles [43-45]. The size of metal nanoparticles synthesized within polyamidoamine (PAMAM) dendrimers is controlled by the number of functional groups in the dendrimer and the structure of the dendrimer matrix (Fig. 1). The number of internal tertiary amine groups dictates the maximum number of metal ions that can be encapsulated within the matrix and, therefore controls the nanoparticle's size [46]. The quasispherical hyperbranched structure of the dendrimer supplies internal cavities for nanoparticle's growth upon reduction of metal ions. The dendrimer also provides a shell that prevents aggregation of the as-grown nanoparticles. By changing the metal ion to dendrimer concentrations ratio, a variety of monometallic and bimetallic nanoparticles in the size range of $1 \mathrm{~nm}$ were synthesized with narrow size distribution [43-45].

$1 \mathrm{~nm}$ sized dendrimer-encapsulated $\mathrm{Rh}$ and $\mathrm{Pt}$ nanoparticles were synthesized and characterized with X-ray Photoelectron spectroscopy (XPS). Analysis of the spectra indicated that $44 \%$ of the $\mathrm{Rh}$ within the $\mathrm{Rh}$ nanoparticles was oxidized, whereas $93 \%$ of the Pt was oxidized in the Pt nanoparticles (Fig. 2). These results demonstrate the direct correlation between the small size of the nanoparticle and their oxidation state.

Dendrimer-encapsulated nanoparticles were immobilized within high surface-area mesoporous silica. Electrostatic interactions and hydrogen bonding between the dendrimer's matrix and the silica support anchored the encapsulated nanoparticles to the support, minimizing any leaching or aggregation of the nanoparticle. The dendrimer-encapsulated nanoparticles were active as catalysts for ethylene hydrogenation reaction without any pretreatment [37]. The catalytic reactivity indicates that even with the encapsulated matrix, most of the nanoparticle's surface area is still accessible for activation of reactant molecules. After a mild reduction process at $423 \mathrm{~K}$ in 76 torr of $\mathrm{H}_{2}$, the catalytic reactivity for ethylene hydrogenation was maximized due to reduction of the oxidized $\mathrm{Rh}$ and $\mathrm{Pt}$ nanoparticles. Preservation of the small particle size and its' catalytic reactivity after the pretreatments demonstrates the high stability of the encapsulated metal. However, pretreatment at higher temperature $(673 \mathrm{~K})$ led to sintering of the nanoparticles, followed by a decrease in their catalytic reactivity.
Fig. 1 Preparation scheme and HR-TEM image of dendrimerencapsulated metallic nanoclusters

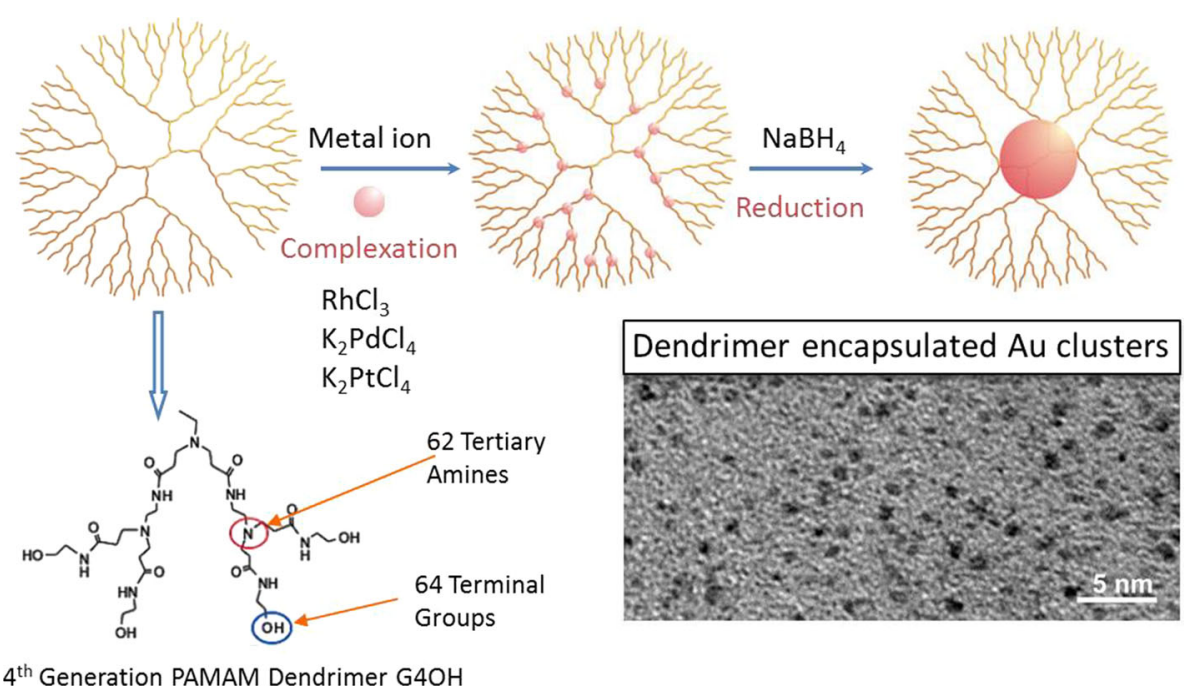


Fig. 2 XPS spectra of dendrimer encapsulated a Rh and $\mathbf{b} \mathrm{Pt}$ nanoparticles. For Rh nanoparticles, $44 \%$ of the Rh was oxidized, whereas $93 \%$ of the $\mathrm{Pt}$ was oxidized for the $\mathrm{Pt}$ nanoparticles

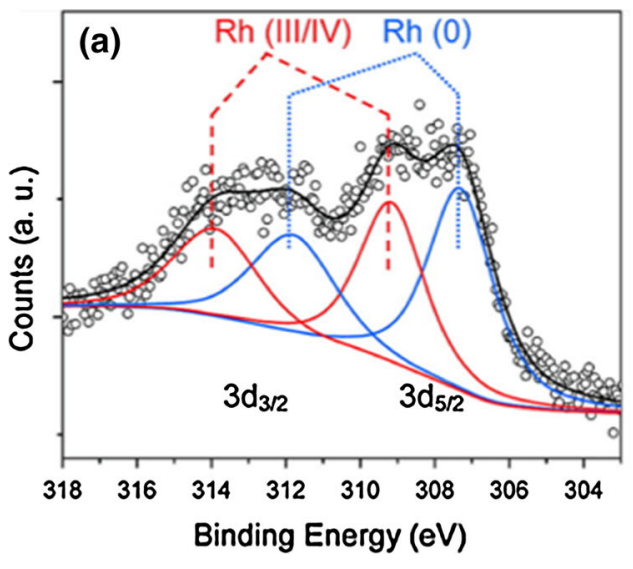

\subsection{Dendrimer-Encapsulated Metallic Nanoparticles as Catalysts for $\pi$-Activation Reactions}

\subsubsection{Catalytic Properties of the Heterogeneous Catalyst}

As suggested by their oxidation state, dendrimer-encapsulated metallic nanoparticles have significant electrophilic character that is similar to that of electron-deficient, latemetal homogeneous catalysts. Following this observation, it was hypothesized that these catalysts would activate new solution-phase carbon-carbon and carbon-heteroatom reactivity that could not have been previously observed with heterogeneous catalysts.

Dendrimer-encapsulated Pt nanoparticles were synthesized, loaded on mesoporous silica and their catalytic reactivity was tested toward a variety of organic transformations [31]. In order to further oxidize and activate the Pt nanoparticles prior to the catalytic reaction, the metallic clusters were exposed to $\mathrm{PhICl}_{2}$; a reagent that acts as a mild oxidizer. Following this pretreatment, the heterogeneous catalysts showed high reactivity towards hydroalkoxylation reactions with yields generally $>99 \%$, which is similar to the reactivity of a homogeneous organometallic Pt complex (Fig. 3). The reaction was performed at $100{ }^{\circ} \mathrm{C}$ with toluene as an organic solvent. The silica-supported dendrimer-encapsulated Pt nanoparticle showed excellent recyclability over multiple cycles after simple filtration, reduction and pretreatment with $\mathrm{PhICl}_{2}$. A sample of silica loaded dendrimer-encapsulated Pt nanoparticle has been recycled four times with a consistent yield of $>90 \%$ under the reported reaction conditions. Following its high reactivity in hydroalkoxylation reaction, the catalyst was tested toward additional $\pi$-bond activation reactions. The highly oxidized nanoparticles catalyzed several cyclization reactions in the solution phase under relatively mild conditions with minimal or no side reactions.

The catalytic reactivity toward the hydroalkoxylation reaction was further enhanced when the Pt cluster in the
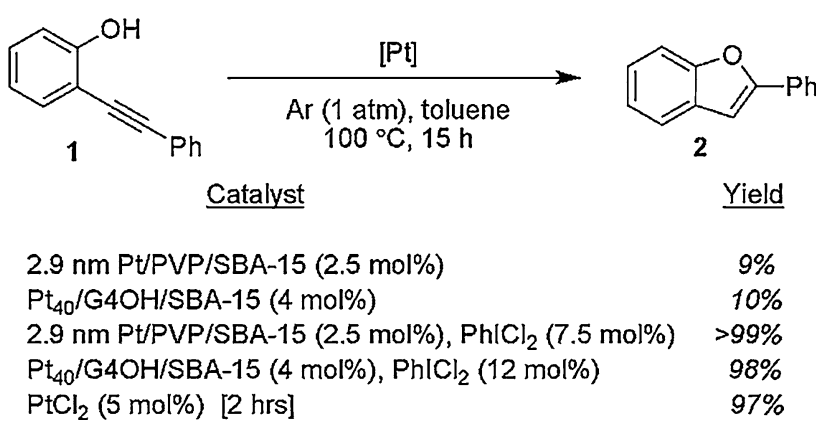

Fig. 3 Hydroalkoxylation of 1 with Pt catalysts. To obtain electrophilic activity from the Pt NPs, pretreatment with the mild oxidant $\mathrm{PhICl}_{2}$ is required. Yields were determined by NMR versus internal standard

dendrimer matrix was replaced with Pd cluster [32]. High reactivity ( $95 \%$ yield) was obtained with reaction time of $4 \mathrm{~h}$ at room temperature with dendrimer-encapsulated $\mathrm{Pd}$ nanoparticles, while only $5 \%$ yield was measured with the homogeneous catalyst (e.g. $\mathrm{PdBr}_{2}$ ) under similar conditions.

\subsubsection{Catalytic Reactivity Under Continuous Flow Conditions}

Since no leaching of catalytically active species was detected from the dendrimer-encapsulated $\mathrm{Pd}$ catalyst during the batch-mode reaction, the catalyst was applied in a fixed bed plug flow reactor to catalyze the same reaction in a continuous-flow mode [32]. The transition from batch to flow mode circumvents the necessity of separating the catalyst from the reaction medium [47]. Furthermore, as long as the highly active heterogeneous catalyst can achieve a full conversion of reactants to products, any further purification of the product is avoided. As shown by the blue dots in Fig. 4a, dendrimer encapsulated Pd nanoparticles, which were pretreated with $\mathrm{PhICl}_{2}$, completed the reactant into product conversion at room temperature. 


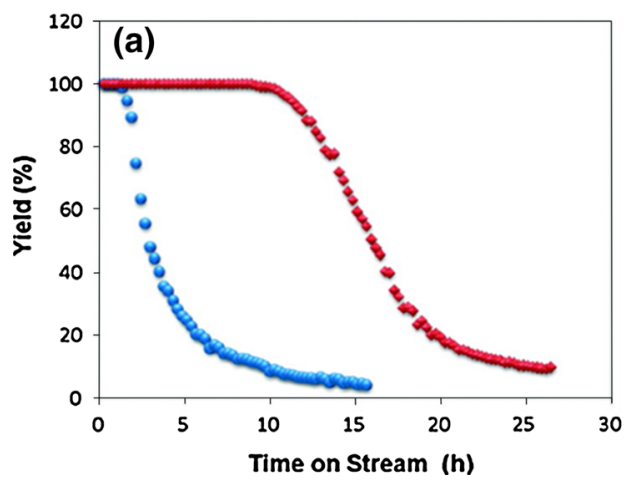

Fig. 4 a Time on stream of product yield catalyzed by dendrimer encapsulated Pd nanoparticles. In the presence of $5 \mathrm{mM} \mathrm{PhICl}_{2}$ in the reaction stream the catalyst was more stable and was deactivated relatively slowly (red diamonds), compared to the case when only

However, the catalytic activity quickly deteriorated to less than $5 \%$ conversion after $15 \mathrm{~h}$. However, when the reaction solution contained $5 \mathrm{mM}$ of $\mathrm{PhICl}_{2}$, the reactivity was restored and $100 \%$ conversion was observed for more than $10 \mathrm{~h}$. The dependence of product yield on reaction time indicates that the catalyst is deactivated faster if no oxidizing agent is present in the solution. When the oxidizing agent is introduced to the catalyst along with the reactant, the deactivated catalysts can be in situ reactivated.

The continuous flow mode reaction is a convenient tool for probing different kinetic parameters, such as the activation energy of the catalytic reactions (Fig. 4b). The reactivity at different reaction temperatures was measured in the flow reactor and from which the apparent activation energy was determined to be $20.0 \mathrm{kcal} / \mathrm{mol}$. In order to measure the activation energy in a batch mode reaction, multiple reactions must be repeated at different temperatures. As a result, any operational error in these reactions will generate inaccuracy in the activation energy. Using a flow reactor, all reaction parameters can be controlled precisely and reproduced easily, while the only variable is the reaction temperature. It should be noted, however, that in order to extract kinetic data of catalytic reactions which are performed in flow reactor, the reactivity should be constant throughout the measurements without any deactivation. In the example that is shown above, the activation energy was analyzed based on the reactivity during the initial $10 \mathrm{~h}$ of the reaction in which no deactivation of the catalyst was detected.

\subsubsection{Stability of the Heterogeneous Catalyst}

Although the remarkable recyclability of the dendrimerencapsulated nanoparticle is a strong indication for its heterogeneity, further verification of the heterogeneous catalysts stability were performed [31]. A three-phase test

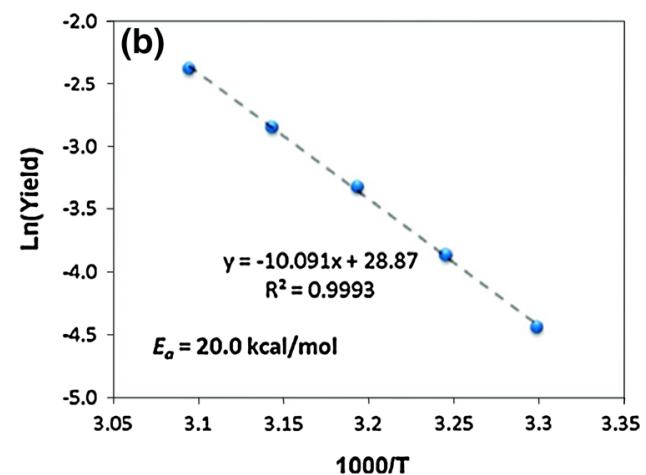

reactant was flowed through the reactor (blue dots). b Activation energy determination of the hydroalkoxylation reaction catalyzed by dendrimer encapsulated Pd nanoparticles using a continuous flow reactor

was conducted with a resin-bound substrate [48, 49]. In the presence of the homogeneous catalyst, $\mathrm{PtCl}_{2}, 26 \%$ conversion of resin-bounds substrate into product was observed. However, the oxidized dendrimer-encapsulated Pt nanoparticle resulted in less than $2 \%$ conversion (Fig. 5a). In addition, transmission electron microscopy images of supported Pt clusters before and after the catalytic reaction did not show any appreciable aggregation or leaching (Fig. 5b).

Furthermore, an experiment was conducted in which the Pt clusters were embedded within a mesoporous silica pellet, to allow for facile removal of the reaction solution from the NP catalyst under reaction conditions (Fig. 5c) [50]. In this case, a solution of starting material, $\mathrm{PhICl}_{2}$ and solvent were added to the catalyst pellet in reactor $\mathbf{A}$ and began to generate the product. After $42 \%$ yield was achieved, the reaction solution in $\mathbf{A}$ was transferred to a new vessel with no catalyst $\mathbf{B}$. The oxidized catalyst pellet remained in A. Following this step a fresh solution of starting material and solvent was added into A. The solution in $\mathbf{A}$ began to convert to product while the solution in B did not react and remained at $42 \%$ yield. These results indicate that the active catalyst is truly heterogeneous. If any homogeneous species were present in the solution, it would have been transferred into vessel $\mathbf{B}$ and an increase in products yield should have been observed. Moreover, the new solution added to $\mathbf{A}$ started to react, showing that the active catalyst has, in fact, remained in $\mathbf{A}$.

\subsection{X-ray Absorption Spectroscopy and Reaction Kinetic Studies of Dendrimer Encapsulated Metallic Nanoparticles}

In order to gain a mechanistic insight of the properties of dendrimer-encapsulated metallic nanoparticles X-ray absorption spectroscopy (XAS), including extended X-ray 
Fig. 5 a A three-phase test to determine the presence of a homogeneous catalyst by leaching or release and capture. b TEM images of dendrimer encapsulated Pt nanoparticles, loaded on mesoporous silica before reaction (left), and after treatment with $\mathrm{PhICl}_{2}$ and reaction with 1 (right). c A reaction vessel $(A)$ was charged with a substrate, a pellet of catalyst and $\mathrm{PhICl}_{2}$ oxidant (blue section). The mixture was stirred and the yield was monitored $(i)$. When the reaction in vessel $A$ reached $42 \%$ yield, the solution was removed and placed into a new vessel $(B)$. A fresh solution of starting material was added to vessel $A$ where the catalyst pellet remained (yellow section). Both vessel $A$ and $B$ were then stirred and monitored (ii) (a)<smiles>O=C(OCc1ccccc1)c1ccccc1C#Cc1ccccc1</smiles>

(b)

\begin{tabular}{ll}
\multicolumn{1}{c}{ Catalyst } & $\frac{\text { Yield }}{26 \%}$ \\
$\mathrm{PtCl}_{2}$ (4 mol\%) & $<2 \%$ \\
$2.9 \mathrm{~nm}$ PUPVP/SBA-15 (2.5 mol\%), $\mathrm{PhICl}_{2}(7.5 \mathrm{~mol} \%)$ & $<2 \%$ \\
$\mathrm{Pt}_{40} / \mathrm{G} 4 \mathrm{OH} / \mathrm{SBA}-15$ (4 mol\%), $\mathrm{PhICl}_{2}(12 \mathrm{~mol} \%)$ & $<2 \%$
\end{tabular}

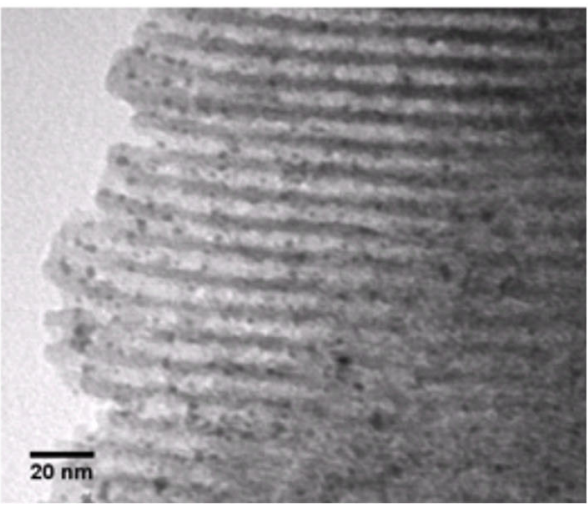

(c)

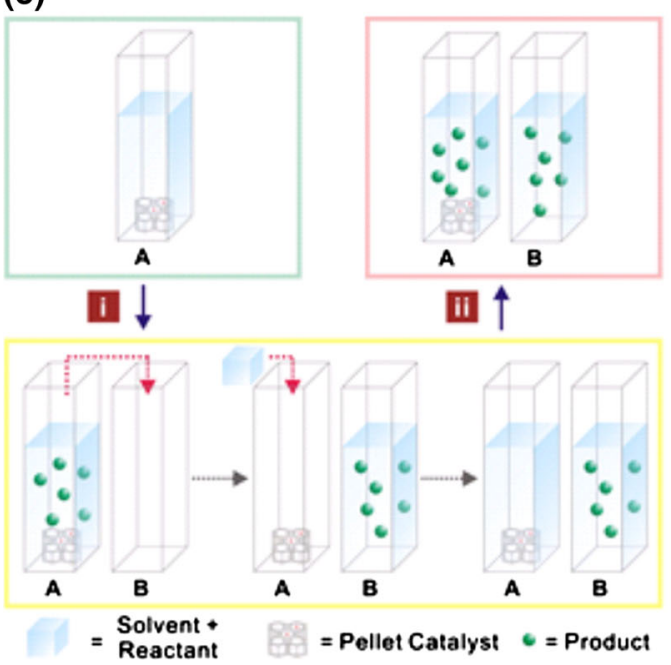

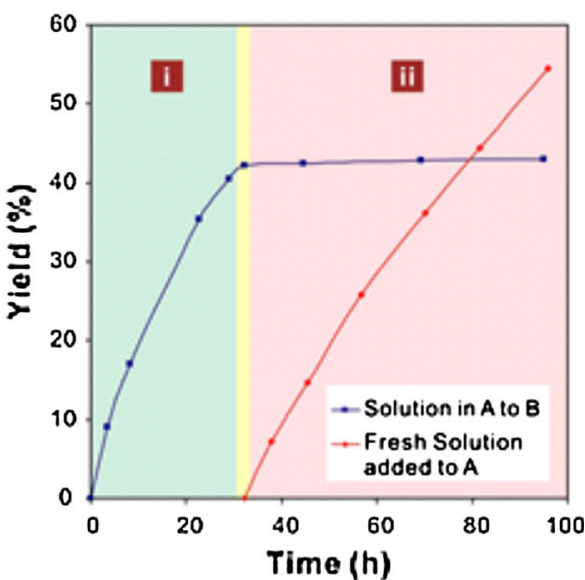

absorption fine structure spectroscopy (EXAFS) and nearedge X-ray absorption fine structure spectroscopy (NEXAFS), were conducted [30]. These measurements provide structural and composition analysis of the catalysts under solution-phase reaction conditions. To analyze composition-reactivity correlations of the $\mathrm{Pt}$ catalyst, samples with varying degree of oxidation were prepared by a sequence of pretreatments such as reduction by $\mathrm{H}_{2}$ and oxidation by $\mathrm{PhICl}_{2}$.

XAS studies showed that the coordination numbers and the oxidation state of $\mathrm{Pt}$ in the dendrimer-encapsulated metal clusters can be easily altered by cycles of reduction and oxidation treatment (Fig. 6a). This effect was correlated to the small size $(1 \mathrm{~nm})$ of the metal clusters. After an initial reduction pretreatment, the Pt-Pt coordination number, derived from EXAFS analysis, was about 5, indicating an average particle size of about $1 \mathrm{~nm}$. Following a subsequent oxidation treatment with $\mathrm{PhICl}_{2}$, the $\mathrm{Pt}-\mathrm{Pt}$ coordination number decreased to about one while the $\mathrm{Pt}-\mathrm{Cl}$ coordination number increased to about 2 .

The second reduction treatment brought the $\mathrm{Pt}-\mathrm{Pt}$ coordination number back to about 4.5 , and the metallic cluster-structure was largely recovered. The changes in oxidation state of the catalyst were also reflected in the fractions of $\mathrm{Pt}(0), \mathrm{Pt}(\mathrm{II})$, and $\mathrm{Pt}(\mathrm{IV})$ species obtained from NEXAFS analysis (Fig. 6b). These results demonstrate that 


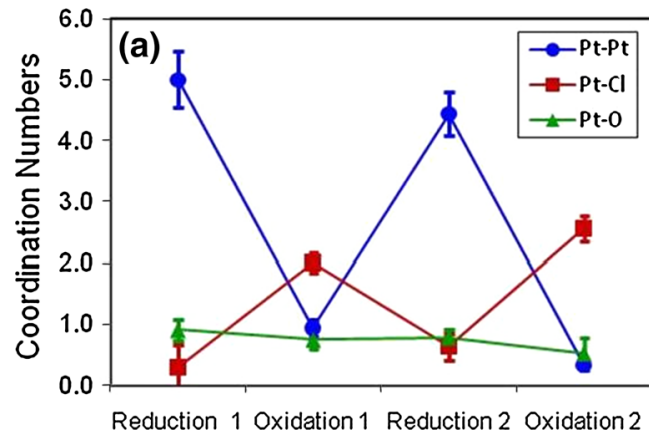

Fig. 6 a Average coordination numbers of $\mathrm{Pt}$ atoms in $\mathrm{SiO}_{2}$ supported after a sequence of hydrogen reduction and $\mathrm{PhICl}_{2}$ oxidation treatments in the toluene derived from EXAFS analysis.

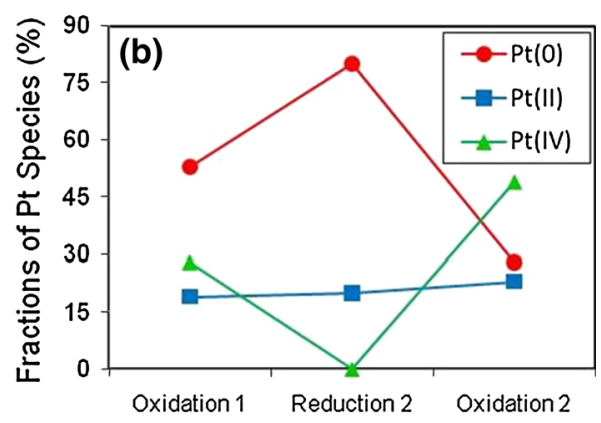

b The fractions of $\mathrm{Pt}(0)$, and $\mathrm{Pt}(\mathrm{II})$ and $\mathrm{Pt}(\mathrm{IV})$ chloride species of the dendrimer-encapsulated $\mathrm{Pt}$ nanoclusters derived from NEXAFS analysis

(a)

(b)

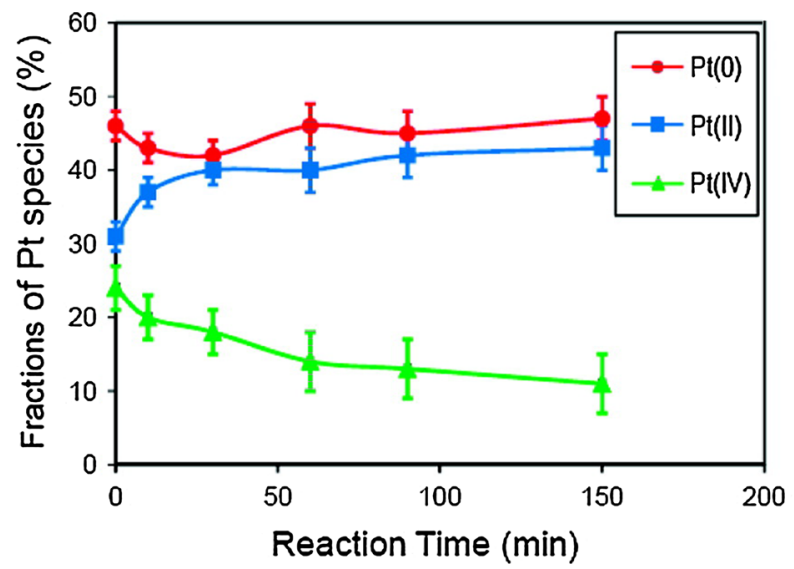

Fig. 7 a Hydroalkoxylation reaction scheme of 2-phenylethynylphenol. b The evolution of Pt species in supported dendrimer-encapsulated Pt clusters catalyst as obtained by NEXAFS measurements under reaction conditions. The $2 \mathrm{wt} \% \mathrm{Pt}$ catalyst was oxidized by

the changes in both coordination numbers and oxidation state of Pt catalyst are reversible during the sequence of reduction and oxidation treatment.

The evolution of the Pt chloride species in the catalyst was monitored by in situ NEXAFS under reaction conditions, in order to correlate the catalyst deactivation process with changes in the fraction of Pt active species (Fig. 7). The reaction kinetics results showed that the conversion rate increased with the fractions of $\mathrm{Pt}(\mathrm{IV})$ species in the oxidized catalyst. Therefore, it was concluded that the $\mathrm{Pt}(\mathrm{IV})$ species was more active than the Pt(II) species. The evolution of the different fractions of Pt species by in situ XAS measurements revealed that the $\mathrm{Pt}(\mathrm{IV})$ species was not stable under reaction conditions, and was partially (c)

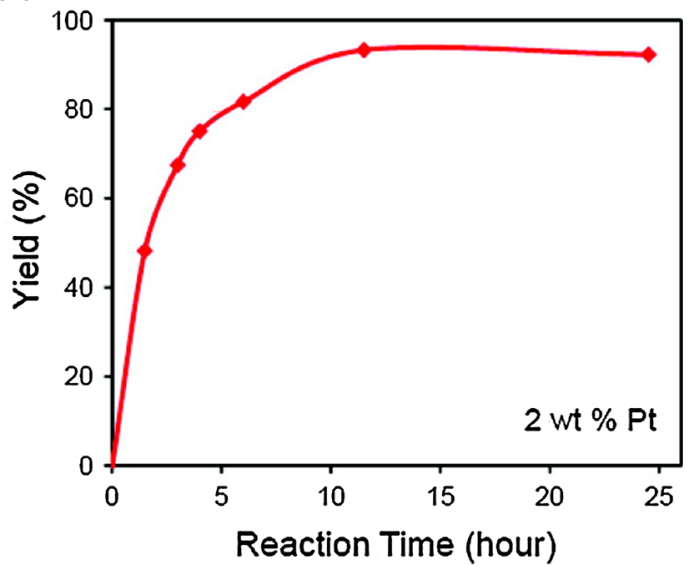

$\mathrm{PhICl}_{2}$ prior to the reaction. c Product yield as a function of time obtained in a batch reaction with 2 wt $\%$ dendrimer encapsulated $\mathrm{Pt}$ clusters loaded on mesoporous $\mathrm{SiO}_{2}$

converted to the $\mathrm{Pt}(\mathrm{II})$ species as the reaction proceeds. The depletion of the fraction of the Pt(IV) species correlates with the catalyst deactivation which was observed in the reaction kinetics study.

The aforementioned reversibility in the cluster's structure suggests that after oxidation Pt ions [both Pt(II) and $\mathrm{Pt}(\mathrm{IV})]$ can be stabilized inside the dendrimer without leaching. The increased stability was correlated to the differences between the hydrophobic solvent (e.g. toluene) and the hydrophilic components of the catalyst including the Pt ion, the PAMAM G4-OH dendrimer, and the silica support. These polar materials have a low solubility in toluene. Thus, as long as this catalyst is used in a nonpolar solvent, the Pt ions would preferentially remain in the polar 
environment within the dendrimer, and the dendrimers would preferentially anchor on the surface of polar silica support.

\subsection{Tuning the Catalytic Diastereoselectivity by Changing the Properties of the Dendrimer Matrix}

Dendrimer-encapsulated Pd and Pt clusters, when oxidized by $\mathrm{PhICl}_{2}$, catalyzed a range of $\pi$-bond activation. These highly active heterogeneous catalysts were generated by the reversible oxidation of metal clusters to ions, which were stabilized by the encapsulating dendrimer. In order to understand the impact of the encapsulating-matrix properties on the product selectivity, dendrimer encapsulated $\mathrm{Au}$ nanoparticles were loaded on mesoporous silica and tested as catalysts for the multiproduct cyclopropanation reaction [29]. In this reaction either cis or trans diastereomers can be formed. While employing the homogenous Au catalysts a cis:trans ratio of 3.5:1 was detected. However, when the homogeneous catalyst was replaced by its heterogeneous analog, the products diastereoselectivity was enhanced by five fold to 16:1. It was hypothesized that the steric effect of the encapsulating matrix favors the formation of the $\mathrm{cis}$ isomer [51]. In order to validate this hypothesis a dendrimer matrix with lower density was synthesized and employed as an encapsulating polymer. With a less crowded matrix the steric effect was reduced and the cis:trans ratio deteriorated to $8: 1$. These results demonstrate that the dendrimer matrix can impact the products distribution, in addition to its role in encapsulating and stabilizing the catalytically active nanoparticles.

To take full advantage of the heterogeneous nature and high reactivity and selectivity of the dendrimer-encapsulated $\mathrm{Au}$ nanoclusters, the catalyst was packed and tested in a flow reactor. Importantly, the high yield and diastereoselectivity of the dendrimer-encapsulated Au catalyst was maintained when the catalytic reaction was transferred from the batch into the flow mode.

\subsection{Controlling the Products Selectivity in Cascade Reactions}

In the section above we demonstrated that diastereoselectivity in cyclopropanation reaction can be controlled by tuning the matrix properties, which is similar to the steric effect of ligands in homogeneous catalyst. Additionally, it is also well known that in homogeneous catalysts the ligands can have a profound impact on the chemoselectivity. As an alternative strategy, we envisioned that chemoselectivity could be achieved with the heterogeneous system by modifying the residence time of the reactants in a flow reactor [29]. In a cascade reaction, an intermediate can be selectively trapped by shortening the residence time, preventing its further reaction. Conversely, it may be possible to maximize the yield of the final rearrangement product by maximizing the residence time of reactants within the flow reactor.

On the basis of this hypothesis, dendrimer-encapsulated $\mathrm{Au}$ catalyst was employed for the sequential cyclopropanation-rearrangement reaction (Fig. 8). The heterogeneous nature of the Au clusters allowed the tuning of product distribution by modifying the flow parameters. By increasing the residence time of the reactants, both the reactivity and the selectivity towards the formation of the secondary products were enhanced. These results demonstrate the potential advantages of heterogenizing homogeneous catalysts and highlight the unique capabilities to direct the reactivity and stereoselectivity of heterogeneous catalysts.

\subsection{High Spatial Resolution Analysis of Cascade Reaction Within a Flow Reactor}

Catalytic reactions along flow reactors can be analyzed by a variety of spectroscopic tools, such as fluorescence, IR, Raman, NMR, and X-ray spectroscopy [39-42]. However, these methods do not have sufficient spatial or spectral resolution for detailed kinetic analysis of the catalytic reaction. In order to gain a detailed mechanistic analysis of catalytic reactions we employed synchrotron-based FTIR microspectroscopy as a tool for mapping catalytic transformations [52]. Two nanometer sized $\mathrm{Au}$ nanoclusters were encapsulated within a dendrimer matrix and loaded on mesoporous $\mathrm{SiO}_{2}$ support [53]. The catalyst was then packed within a flow microreactor and its reactivity was tested toward the cascade reaction of dihydropyran formation (Fig. 9).

Propargyl vinyl ether $\mathbf{1}$ was catalytically rearranged by the Au catalyst to the primary product, allenic aldehyde $\mathbf{2}$. Activation of the primary product by the Au catalyst was followed by nucleophilic attack of butanol- $d_{10}$, which induced the formation of the secondary product, acetal 3. In this cascade reaction, which was previously catalyzed by homogeneous $\mathrm{Au}$ complexes, each of the reactants and products show distinguishable IR signatures. As a consequence, the reactants-into-products evolution can be precisely monitored with IR spectroscopy.

At high flow rate $(1 \mathrm{~mL} / \mathrm{h})$ of the reactants (Fig. 9a) only few changes were observed in the lower energy regime $\left(1,150-2,000 \mathrm{~cm}^{-1}\right)$. A new absorption peak was detected at $1,720 \mathrm{~cm}^{-1}$, indicating the formation of the primary product, allenic aldehyde $\mathbf{2}$. Interestingly, a maximum in the absorption peak of the primary product was detected at $0.2 \mathrm{~mm}$, before setting into steady absorption amplitude at $0.6 \mathrm{~mm}$. This gradual decrease in aldehyde 

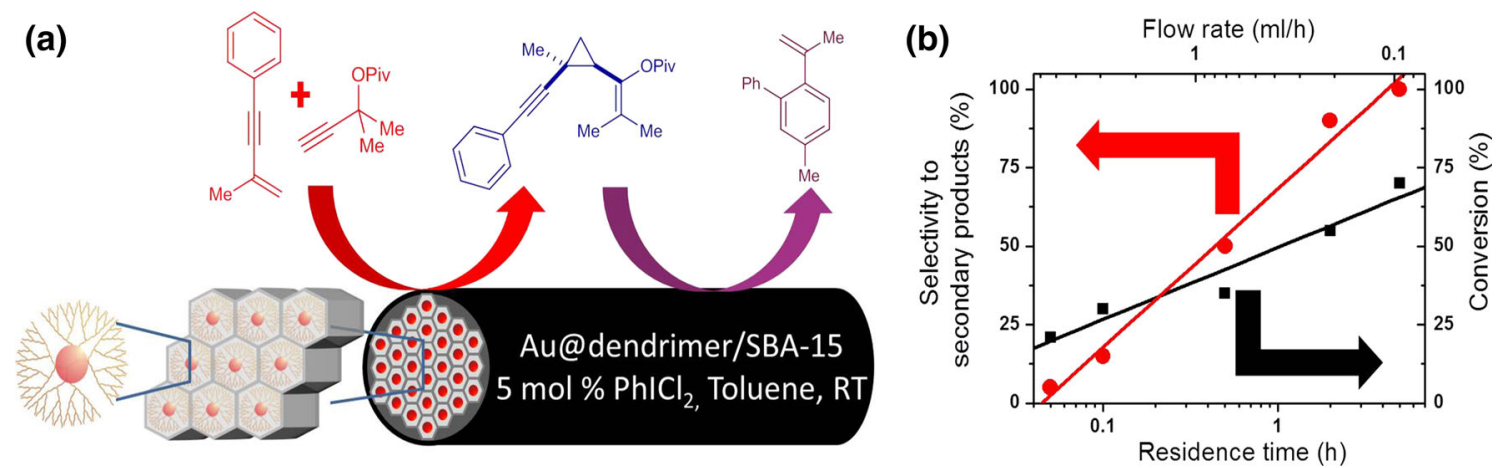

Fig. 8 a Scheme of gold catalyzed cascade cyclopropanation rearrangement reaction under continuous flow conditions. b Conversion and selectivity of the cascade cyclopropanation reaction as function of reactants residence time. The conversion (black squares) and selectivity (red squares) toward the formation of secondary products were enhanced along with the increase in the reactants residence time
Fig. 9 IR absorption microspectroscopy scans along the flow reactor with reactants flow rates of 1 (a) and 0.2 (b) $\mathrm{mL} / \mathrm{hr}$. Markers were added to direct the eye toward the changes in the IR spectra at different flow rates. A scheme of the IR beam scan along the flow reactor $(\mathbf{c})$
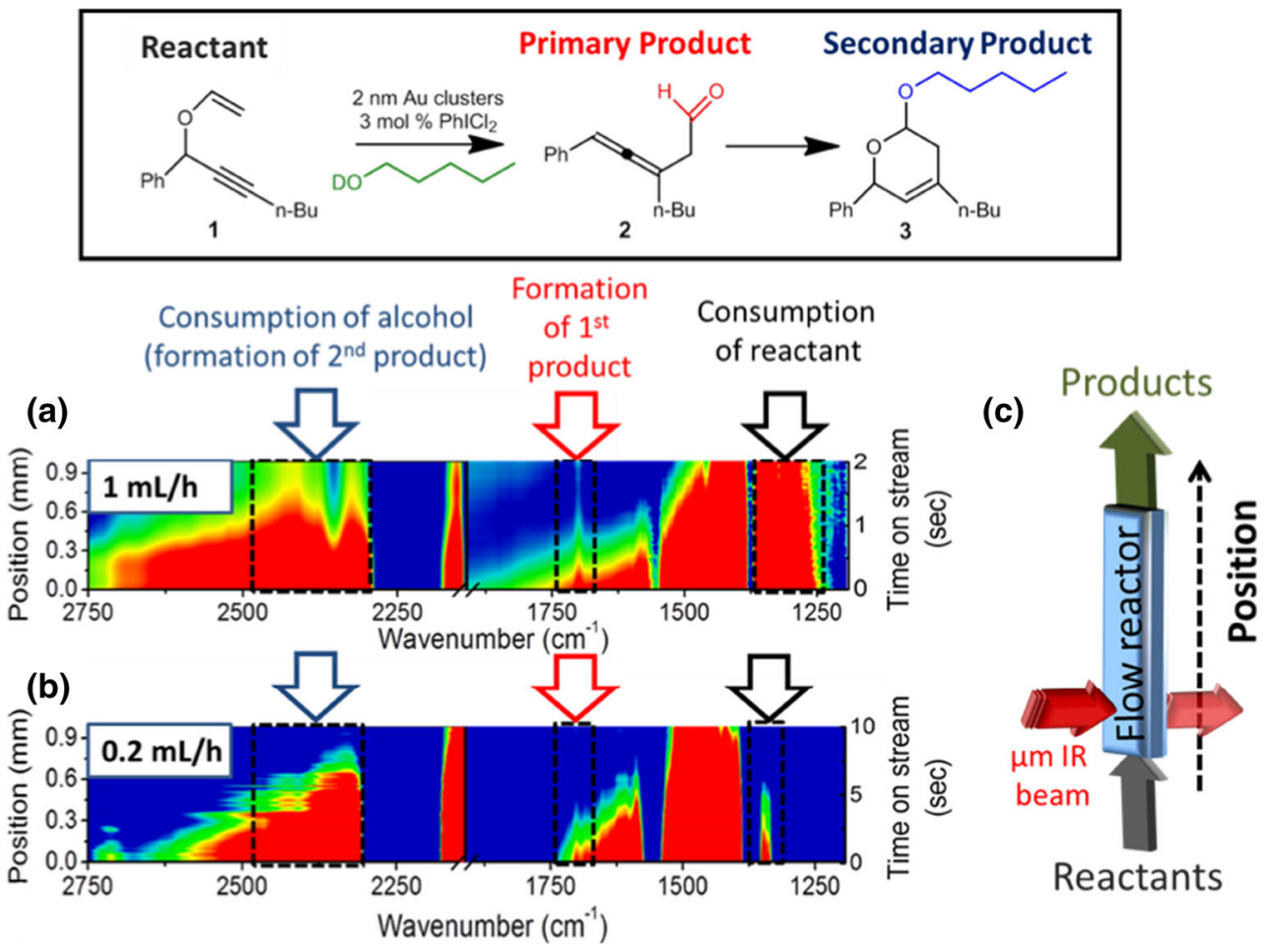

absorption is correlated to partial transformation of the primary product into the secondary product, acetal 3. A decrease was obtained in the IR absorption lines which are correlated to vinyl ether $\mathbf{1}(1,200-1,400$ and $1,600-1,700 \mathrm{~cm}^{-1}$ ), indicating that fraction of the reactants was consumed within this residence time. Additional changes were observed in the higher energy range $\left(2,150-2,750 \mathrm{~cm}^{-1}\right)$, which indicate the consumption of butanol- $d_{10}$ and the partial formation of secondary products under these conditions.

Higher yield was obtained when the residence time of the reactants was increased fivefold (Fig. 9b), as detected by the complete loss of the reactant's absorption peaks at
$1,150-1,250 \mathrm{~cm}^{-1}$ after $0.6 \mathrm{~mm}$. Though primary product 2 was initially formed, after $0.4 \mathrm{~mm}$ its IR signature was fully lost, indicating the primary into secondary product transformation. The formation of the secondary product, acetal $\mathbf{3}$, is demonstrated as well by the consumption of butanol- $d_{10}$ which is observed by the gradual decrease in the amplitude of O-D absorption peak at 2,300$2,650 \mathrm{~cm}^{-1}$ and its disappearance after $0.8 \mathrm{~mm}$. It can be concluded that by decreasing the flow rate, continuous consumption of vinyl ether $\mathbf{1}$ was followed by formation of the primary product, allenic aldehyde $\mathbf{2}$, which further reacted with the catalyst and the nucleophile alcohol to form the secondary product, acetal $\mathbf{3}$. The ratio between the 
primary and secondary products was correlated to the flow rate of the reactants.

A detailed kinetic analysis of the catalytic reaction was performed by integrating the IR absorption peaks of the reactants and products as a function of time on stream and location along the flow reactor. The IR absorption peaks were converted to concentration values $(\mathrm{mmol} / \mathrm{L})$ according to GC analysis of the solution before and after the catalytic reaction (Fig. 10).

The kinetic evolution of the reaction was divided into few stages (Fig. 10a, b). In the initial phase of the reaction, the concentration of reactant $\mathbf{1}$ gradually decreased due to formation of primary product $\mathbf{2}$. During this incubation time, no formation of secondary product $\mathbf{3}$ or consumption of butanol- $d_{10}$ were detected. Through the second part of the reaction the two reactants (vinyl ether 1 and butanol- $d_{10}$ ) and the primary product (allenic aldehyde 2 ) were consumed with concomitant formation of the secondary product, acetal 3. Zero order kinetics with similar reaction rate coefficients were measured for vinyl ether $\mathbf{1}$ and butanol- $d_{10}$. First order kinetics was fitted to the primary product allenic aldehyde $\mathbf{2}$. At this stage of the reaction the local concentration of the two reactants (vinyl ether $\mathbf{1}$ and butanol- $d_{10}$ ) saturated the catalyst and induced zero order kinetics.

At slower flow rate of $0.2 \mathrm{ml} / \mathrm{hr}$ (Fig. 10b) an additional third phase of the reaction was obtained in which the concentration of the reactants was low enough to facilitate the conversion from zero to first order kinetics for both reactants. At this stage, a continuous low, steady state concentration of allenic aldehyde $\mathbf{2}$ was detected, demonstrating its pivotal role as an intermediate for the formation of the secondary product, acetal $\mathbf{3}$. These results verified that the rate-determining step in this reaction is the transformation of vinyl ether $\mathbf{1}$ to allenic aldehyde 2 . The mapping of multistep catalytic process enabled an in-depth analysis of the mechanism of catalytic reactions. These in situ measurements can be widely utilized to probe and improve the catalytic yield and products selectivity.
2.7 Utilization of Asymmetric Environment that Encapsulates Metallic Nanoparticles as a Source for Enantioselectivity

The development of sophisticated nanostructures for unique catalytic selectivity is widely used in nature [54, 55]. For example, three-dimensional structure of enzyme can produce high catalytic enantioselectivity. It was hypothesized that in mesoscale structures high enantioselectivity could be achieved by surrounding the catalytic site with a chiral environment (Fig. 11).

In order to study this hypothesis, heterogeneous Au clusters were synthesized within chiral self-assembled layers and were tested as catalyst for asymmetric cyclopropanation reaction $[28,56]$. Complexation of $\mathrm{Au}$ ions within the self-assembled layers enabled the formation of $\mathrm{Au}$ clusters that are encapsulated within the chiral environment. The Au catalyst was activated by its' oxidation with $\mathrm{PhICl}_{2}$. Under these conditions, the heterogeneous $\mathrm{Au}$ catalyst was activated toward intermolecular and intramolecular cyclopropanation reactions with up to 51 and $30 \%$ enantiomeric excess, respectively. In the case of the intermolecular cyclopropanation reaction, the enantioselectivity was coupled with high diastereoselectivity.

The oxidation state of the catalyst was continuously measured with NEXAFS spectroscopy under reaction conditions while product formation was analyzed by GCMS. Direct correlation was observed between the reactivity of the heterogeneous catalyst and high concentration of $\mathrm{Au}(\mathrm{III})$ species. The catalytic reactivity was quenched following the reduction of $\mathrm{Au}(\mathrm{III})$ into $\mathrm{Au}(0)$. This correlation indicated that highly oxidized $\mathrm{Au}(\mathrm{III})$ are the active species in this reaction. Spectroscopic analysis revealed that hydrogen-bonding network was formed within the molecules construct the self-assembled layers as the number of amino acids in these molecules was increased above one. The formation of hydrogen-bonding network within the polymeric matrix stabilized the self-assembled layers.
Fig. 10 Analysis of distribution of reactants and products as a function of location along the flow reactor at a flow rate of 1 (a) and $0.2(\mathbf{b}) \mathrm{mL} / \mathrm{h}$. The absorption values of the reactants and products were integrated from the matching IR absorption peak areas and converted to concentration based on GC analysis of the solution. The dotted lines indicate the different stages of the catalytic reaction
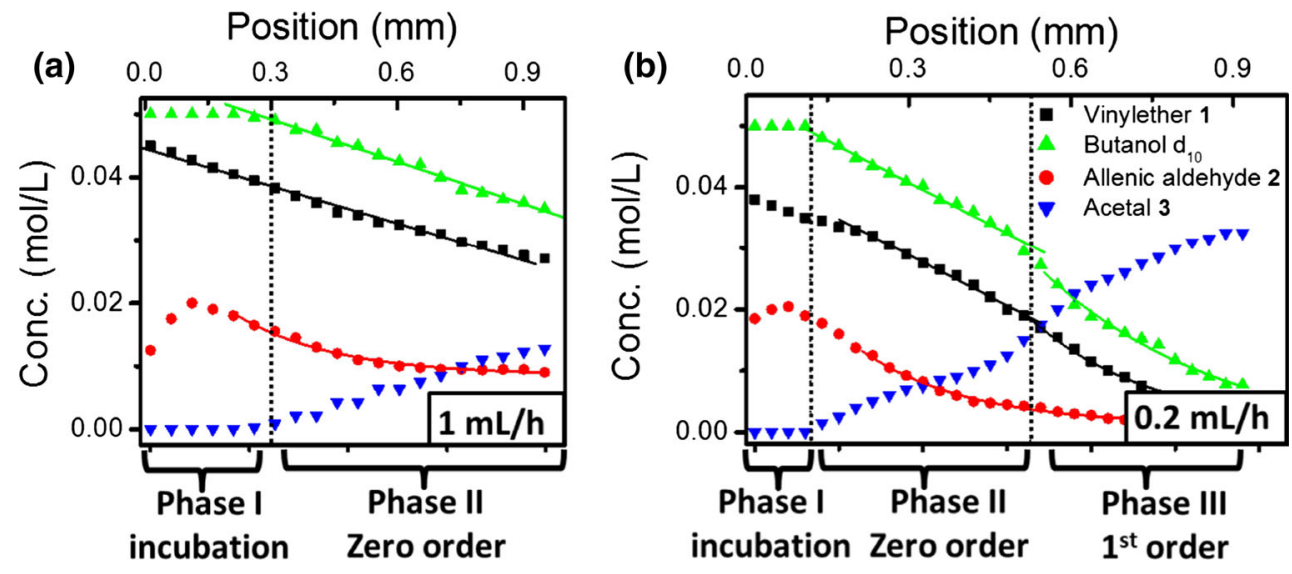
Fig. 11 a Reaction scheme of cyclopropane formation. b Cartoon of the mesoscale catalyst, constructed of $\mathrm{Au}$ nanoparticles surrounded by chiral molecules which were grafted on mesoporous $\mathrm{SiO}_{2}$ support. c The enantioselectivity was enhanced by threefold by increasing the number of amino acid in the grafted peptides from one to two

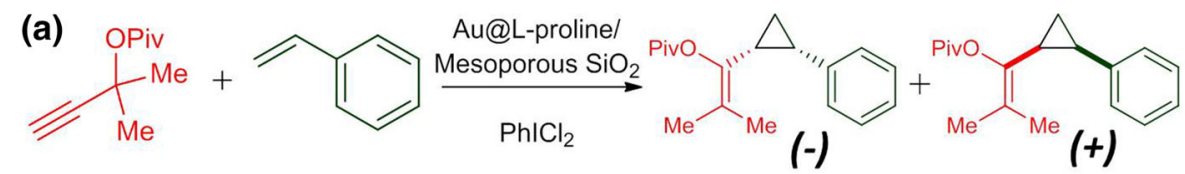

(b)

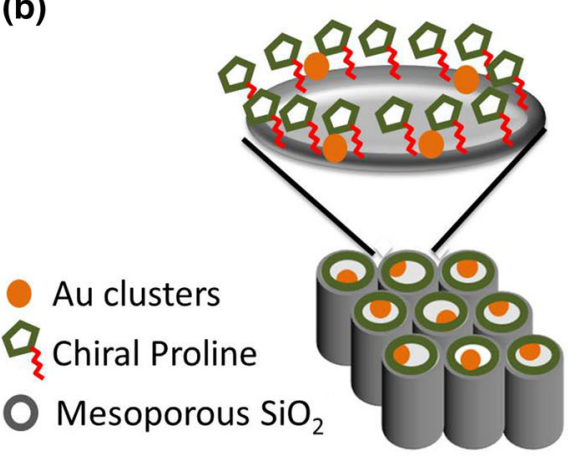

(c)
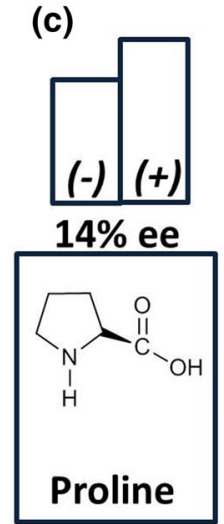

$(-)(+)$ $51 \%$ ee

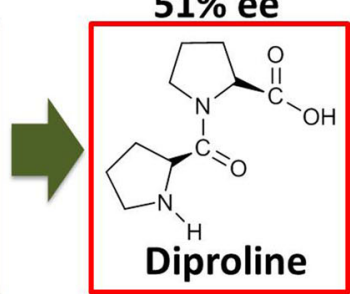

The intermolecular and intramolecular hydrogen bonding of the peptides formed better encapsulation of the metal surface and therefore to some extent can modify the alignment of reactant molecules as they approach to the catalytically active Au ions.

These results demonstrate the advantage of mesoscale catalysts that are assembled from two (or more) different building blocks for the formation of architecturally designed catalysts. The combination of metallic nanoclusters and specifically-designed encapsulating matrix enabled the preparation of both reactive and enantioselective heterogeneous catalysts. Unlike other asymmetric heterogeneous catalysts in which ligand-reactant and ligandcatalyst interactions are essential for enantioselective reactions, while employing the amino-based chiral encapsulating layer the enantioselectivity was solely induced by the chirality of the matrix itself.

\section{Conclusion and Outlook}

In this review paper we introduced our recent results in studying the interface between heterogeneous and homogeneous catalysis [57]. The initial key observation that initiated this study was that by decreasing the nanoparticles size beyond $1 \mathrm{~nm}$, its electronic properties and therefore also its catalytic properties can become similar to that of homogeneous catalyst. Following this observation, small metallic nanoparticle were activated toward a wide array of catalytic reactions that were previously activated by homogeneous catalysts.

The dendrimer matrix that encapsulates the nanoparticles has a crucial role in stabilizing the catalytically active nanoparticles under reaction conditions. It was demonstrated that the main source of catalysts stabilization are repulsion forces between the hydrophilic catalyst and hydrophobic solvent. Changes in the properties of the solvent or the catalyst induced substantial leaching of metal ions to the solution phase and loss of the heterogeneous character of the catalyst.

In addition to stabilization of the metal cluster, the matrix can be utilized as catalytic promoter and tune the catalytic selectivity. By changing the steric properties of the matrix the products diastereoselectivity ratio can be optimized. It was also demonstrated that the dendrimer matrix can be replaced with a self-assembled layer of asymmetric peptides that induced high catalytic stability and enantioselectivity. These results uncover the vital potential role of the cluster's surroundings as a co-catalyst.

A variety of in situ spectroscopy measurements were conducted in order to characterize the properties of the metallic nanoparticles under reaction conditions. X-ray absorption spectroscopy verified that the catalytic reactivity of dendrimer-encapsulated metallic nanoparticles is correlated to the formation and stabilization of highly oxidized metal ions within the matrix. The catalytic reactivity was quenched following the reduction of the active ions back into nanoparticles.

Micrometer sized IR beam was employed in order to map the reactants into products transformation within a flow microreactor which was packed with silica-supported dendrimer-encapsulated Au nanoclusters. The high-resolution IR microspectroscopy tracked the formation of different intermediates along the flow reactor and analyzed their role during the catalytic process. X-ray microspectroscopy measurements correlated between the catalytic reactivity along the microreactor and the concentration of highly oxidized metal ions. 
The powerful combination of synthesis, catalysis and in situ spectroscopy provides invaluable data that correlates between the electronic and geometric properties of the catalyst and its reactivity and selectivity. In-situ spectroscopy is vital for uncovering the dynamic properties of the catalyst under reaction conditions and revealing the mechanism that govern and direct these catalytic reactions [58].

Throughout this review paper we discussed the important role of the environment that surrounds the metallic nanoparticle in stabilizing, activating and optimizing the reactivity of the catalytic surface. We demonstrated that the dendrimer matrix that encapsulates the metallic nanoparticle enables the activation of heterogeneous catalyst toward reactions that were previously catalyzed by homogeneous catalysts. Looking ahead into the field of heterogeneous catalysis we can point out two directions that can be evolved on the basis of the above-mentioned conclusions. One approach is the development of highly stable catalytic mesostructures in which the polymeric matrix that encapsulates the metal surface is substituted with a metaloxide support that can act as a co-catalyst [59-61]. The metal-oxide support can (i) tune the electronic properties of the metal and (ii) act as a co-catalyst and directly activate the reactants during the catalytic process. Unlike the polymer-encapsulated nanoparticle which is not stable under harsh conditions (highly acidic or basic environment and high temperatures), the metal-oxide matrix is expected to be stable even under these conditions. As a consequence, it is expected that this mesoscale system can be suitable catalyst for high activation-energy reactions.

Another field of research that goes beyond the homogenous-heterogeneous interface is the endeavor to bridge between heterogeneous and enzymatic catalysis. Few publications have demonstrated that enzyme can be combined with homogeneous catalysts in activating cascade reactions [62-64]. It was also demonstrated that enzymes can be encapsulated within a solid matrix without loosing their high catalytic specificity [65]. These results indicate that it would be plausible to use encapsulated enzymes as heterogeneous catalysts that will activate cascade reactions along with the metal catalyst. This specifically-designed catalyst, that combines enzymatic and metallic properties, may lead to unique product selectivity that could not be attained with the heterogeneous catalyst.

Acknowledgments This work was supported by the Director, Office of Basic Energy Sciences, Materials Science and Engineering Division and the Division of Chemical Sciences, Geological and Biosciences of the U.S. Department of Energy under Contract No. DEAC02-05CH11231. The X-ray absorption and Infrared microspectroscopy studies were performed at the Advanced Light Source, a DOE User facility of the Office of Science, Office of Basic Energy Sciences.

\section{References}

1. Somorjai GA (2004) Nature 430:730

2. Bell AT (2003) Science 299:1688-1691

3. Corma A, Garcia H (2008) Top Catal 48:8-31

4. Smejkal T, Breit B (2008) Angew Chem Int Ed 47:311-315

5. Uraguchi D, Ueki Y, Ooi T (2009) Science 326:120-123

6. Gorin DJ, Toste FD (2007) Nature 446:395-403

7. An K, Somorjai GA (2012) Chemcatchem 4:1512-1524

8. Na K, Zhang Q, Somorjai GA (2014) J Clust Sci 25:83-114

9. Zhang H, Jin MS, Xiong YJ, Lim B, Xia YN (2013) Acc Chem Res 46:1783-1794

10. Cuenya BR (2010) Thin Solid Films 518:3127-3150

11. Astruc D, Lu F, Aranzaes JR (2005) Angew Chem Int Ed 44:7852-7872

12. Gross E, Krier JM, Heinke L, Somorjai GA (2012) Top Catal 55:13-23

13. Chabanas M, Baudouin A, Coperter C, Basset JM (2001) J Am Chem Soc 123:2062-2063

14. Corma A, Garcia H, Xamena FXL (2010) Chem Rev 110:46064655

15. Leusa K, Liub Y, Meledinac M, Turnerc S, Tendelooc G, Van Der Voorta P (2014) J Catal 316:201-209

16. Larabi C, Nielsen PK, Helveg S, Thieuleux C, Johansson FB, Brorson M, Quadrelli EA (2012) ACS Catal 2:695-700

17. Chizallet C, Lazare S, Bazer-Bachi D, Bonnier F, Lecocq V, Soyer E, Quoineaud A, Bats N (2010) J Am Chem Soc 132:12365-12377

18. Jeong KS, Go YB, Shin SM, Lee SJ, Kim J, Yaghi OM, Jeong N (2011) Chem Sci 2:877-882

19. Hiroyuki M, Ryosuke M, Yoji M, Kobayashi S (2007) Angew Chem Int Ed 46:4151-4154

20. Celine L, Takeshi I, Hiroyuki M, Matsubara R, Kobayashi S (2008) Adv Syn Cat 350:1996

21. Ryo A, Kobayashi S (2009) Chem Rev 109:594-642

22. Jean-Francois S, Hiroyuki M, Kobayashi S (2011) J Am Chem Soc 133:18550-18553

23. Carrettin S, Guzman J, Corma A (2005) Angew Chem Int Ed 44:2242-2245

24. Carrettin S, Concepcion P, Corma A, Nieto JML, Puntes VF (2004) Angew Chem Int Ed 43:2538-2540

25. Guzman J, Carrettin S, Corma A (2005) J Am Chem Soc 127:3286-3287

26. Guzman J, Carrettin S, Fierro-Gonzalez JC, Hao Y, Gates BC, Corma A (2005) Angw Chem Int Ed 44:4778-4781

27. Gross E, Somorjai GA (2013) Top Catal 56:1049-1058

28. Gross E, Liu JH, Alayoglu S, Marcus MA, Fakra SC, Toste FD, Somorjai GA (2013) J Am Chem Soc 135:3881-3886

29. Gross E, Liu JHC, Toste FD, Somorjai GA (2012) Nat Chem 4:947-952

30. Li YM, Liu JHC, Witham CA, Huang WY, Marcus MA, Fakra SC, Alayoglu P, Zhu ZW, Thompson CM, Arjun A, Lee K, Gross E, Toste FD, Somorjai GA (2011) J Am Chem Soc 133:13527-13533

31. Witham CA, Huang WY, Tsung CK, Kuhn JN, Somorjai GA, Toste FD (2010) Nat Chem 2:36-41

32. Huang WY, Liu JHC, Alayoglu P, Li YM, Witham CA, Tsung CK, Toste FD, Somorjai GA (2010) J Am Chem Soc 132:16771-16773

33. Yoon B, Hakkinen H, Landman U, Worz AS, Antonietti JM, Abbet S, Judai K, Heiz U (2005) Science 307:403-407

34. Boffa A, Lin C, Bell AT, Somorjai GA (1994) J Catal 149:149-158

35. Landman U, Yoon B, Zhang C, Heiz U, Arenz M (2007) Top Catal 44:145-158 
36. Valden M, Lai X, Goodman DW (1998) Science 281:1647-1650

37. Huang W, Kuhn JN, Tsung CK, Zhang Y, Habas SE, Yang P, Somorjai GA (2008) Nano Lett 8:2027-2034

38. Lykakis IN, Psyllaki A, Stratakis M (2011) J Am Chem Soc 133:10426-10429

39. Lamberti C, Zecchina A, Groppo E, Bordiga S (2010) Chem Soc Rev 39:4951-5001

40. Weckhuysen BM (2009) Angew Chem Int Ed 48:4910-4943

41. Zaera F (2012) Chem Rev 112:2920-2986

42. Weckhuysen BM (2002) Chem Comm 2:97-110

43. Albiter MA, Crooks RM, Zaera F (2010) J Phys Chem Lett $1: 38-40$

44. Niu YH, Yeung LK, Crooks RM (2001) J Am Chem Soc 123:6840-6846

45. Daniel MC, Astruc D (2004) Chem Rev 104:293-346

46. Borodko Y, Thompson CM, Huang W, Yildiz HB, Frei H, Somorjai GA (2011) J Phys Chem C 115:4757-4767

47. Newman SG, Jensen KF (2013) Green Chem 15:1456-1472

48. Collman JP, Kosydar KM, Bressan M, Lamanna W, Garrett T (1984) J Am Chem Soc 106:2569-2579

49. Rebek J, Gavina F (1974) J Am Chem Soc 96:7112-7114

50. Sheldon RA, Wallau M, Arends IWCE, Schuchardt U (1998) Acc Chem Res 31:485-493

51. Johansson MJ, Gorin DJ, Staben ST, Toste FD (2005) J Am Chem Soc 127:18002-18003

52. Jones CW, Tao F, Gerlamd MV (2012) ACS Catal 2:2444-2445

53. Gross E, Shu XZ, Alayoglu S, Bechtel HA, Martin MC, Toste FD, Somorjai GA (2014) J Am Chem Soc 136:3624-3629
54. Solomon EI, Brunold TC, Davis MI, Kemsley JN, Lee SK, Lehnert N, Neese F, Skulan AJ, Yang YS, Zhou J (2000) Chem Rev 100:235-349

55. Merkx M, Kopp DA, Sazinsky MH, Blazyk JL, Muller J, Lippard SJ (2001) Angew Chem Int Ed 40:2782-2807

56. Wang Y-M, Lackner AD, Toste FD (2014) Acc Chem Res 47:889-901

57. Gross E, Somorjai GA (2014) Top Catal 57:812-821

58. Somorjai GA, Park JY (2008) Angew Chem Int Ed 47:9212-9228

59. Baker LR, Kennedy G, Van Spronsen M, Hervier A, Cai XJ, Chen SY, Wang LW, Somorjai GA (2012) J Am Chem Soc 134:14208-14216

60. Kennedy G, Baker LR, Somorjai GA (2014) Angew Chem Int Ed 53:3405-3408

61. An K, Alayoglu S, Musselwhilte N, Plamthottam G, Malaet G, Lindeman AE, Somorjai GA (2013) J Am Chem Soc 135:1668916696

62. Denard CA, Huand H, Bartlett MJ, Lu L, Yichen T, Zhao H, Hartwig JF (2014) Angew Chem Int Ed 126:475-479

63. Denard CA, Hartwig JF, Zhao H (2013) ACS Cat 12:2856-2864

64. Wang ZJ, Clary KN, Bergman RG, Raymond KN, Toste FD (2013) Nat Chem 5:100-103

65. Engstron K, Johnston EV, Verho O, Gustafson KPJ, Shakeri M, Tai CW, Backvall JE (2014) Angew Chem Int Ed 52:1425614260 